\title{
L'épidémiologie basée sur les eaux usées, un outil innovant pour le suivi sanitaire des populations : l'exemple du réseau Obépine, sentinelle de l'épidémie de Covid-19
}

\author{
Wastewater-based epidemiology, an innovative tool for population health monitoring: \\ example of the Obépine network, sentinel of the Covid-19 outbreak
}

L. MOULIN ${ }^{1 *}$, M. BONI ${ }^{2}$, N. CLUZEL ${ }^{3}$, P. GARY 4 , S. LE GUYADER ${ }^{4}$, M. LEVERT ${ }^{5}$, Y. MADAY ${ }^{6}$, V. MARECHAL? J.-M. MOUCHEL ${ }^{5}$, S. WANG ${ }^{3}$, S. WURTZER'

\author{
Eau de Paris - DRDQE, R\&D - Ivry-sur-Seine \\ Institut de recherche biomédicale des armées - Microbiology \& Infectious diseases - Virology unit - Brétigny-sur-Orge \\ ${ }^{3}$ Sorbonne Université - Maison des modélisations ingénieries et technologies (Summit) - Paris \\ Institut français de recherche pour l'exploitation de la mer (lfremer) - Laboratoire de microbiologie LSEM/SG2M - Nantes \\ UMR Metis 7619 - Sorbonne Université - CNRS - École pratique des hautes études (EPHE) - Paris \\ Sorbonne Université - CNRS - Laboratoire Jacques-Louis Lions (LJLL) - Institut universitaire de France - Paris \\ Sorbonne Université - Inserm U938 - Centre de recherche Saint-Antoine - Paris
}

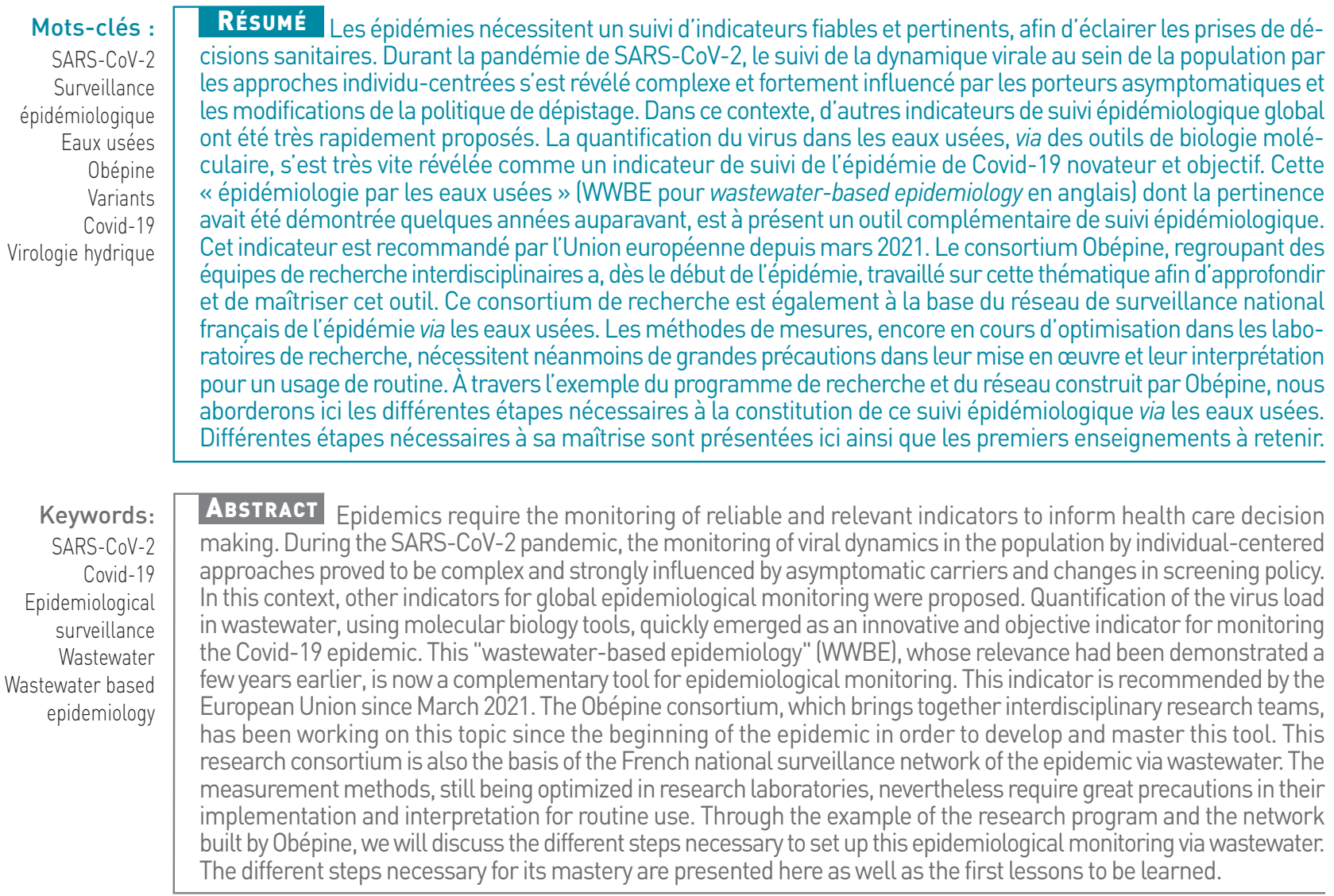

\section{L'analyse des eaux usées : un indicateur supplémentaire pour suivre les épidémies}

1.1. Un concept proposé il y a de nombreuses années

L'analyse des eaux usées pour suivre certaines épidémies humaines n'est pas un concept nouveau et il est utilisé

*Auteur correspondant - Courriel : laurent.moulin@eaudeparis.fr par la communauté scientifique depuis les années 1930, dans certains cas, afin par exemple de suivre la résurgence de la poliomyélite ou l'efficacité des plans de vaccination dans certaines régions [WURTZER et al., 2021a]. La mise au point d'outils de biologie moléculaire de plus en plus performants permet à présent de mettre en place un suivi de la dynamique d'une épidémie via une mesure de la concentration du génome viral. À ce 
titre, le consortium Obépine, regroupant plusieurs équipes françaises de recherche interdisciplinaires, a mis en place sur demande du ministère de la Recherche un réseau de surveillance des eaux usées permettant un suivi de la dynamique épidémique du SARS-CoV-2. Nous expliquerons dans cet article la mise en place de ce réseau, mais également les différents points techniques qui nous semblent important de maitriser pour s'assurer de la qualité et de la pertinence du suivi.

Dès 2015, divers projets de R\&D ont permis de montrer la validité du concept de surveillance quantitative des épidémies. Par exemple, des études réalisées dans le cadre d'un projet de recherche sur les virus entériques au sein du programme de recherche interdisciplinaire sur l'environnement Piren Seine, sur la fréquence des gastro-entérite aiguës [PREVOST et al., 2015b] ont mis en évidence la corrélation entre l'incidence des gastroentérites et la concentration des principaux virus entériques dans les eaux usées dans un bassin versant. De même, le séquençage de l'ensemble des norovirus dans les eaux usées a permis de mettre en évidence certains génotypes viraux, peu ou pas retrouvés dans les échantillons hospitaliers [PREVOST et al., 2015a]. Lors d'une épidémie d'hépatite $E$ sur une île de l'ouest de la France, un lien direct a été établi entre le nombre de personnes infectées et la détection du virus dans les eaux usées [MIURA et al., 2016]. Ces travaux ont participé à la mise en évidence de l'intérêt de suivre les eaux usées par des méthodes moléculaires pour mieux comprendre les épidémies.

\subsection{Intérêt et pertinence de l'analyse des eaux usées pour le suivi de l'épidémie Covid-19}

Ces outils ont été très rapidement appliqués au virus SARS-CoV-2 en France [WURTZER et al., 2020] et à travers le monde et "l'épidémiologie via les eaux usées" (WWBE pour wastewater based epidemiology en anglais) est alors devenue un outil complémentaire de suivi épidémique de la Covid-19, jusqu'à être recommandée par l'Union européenne en mars 2021.

En effet, l'épidémie de Covid-19 a très vite mis en évidence certaines difficultés liées aux indicateurs épidémiologiques classiques (basés sur l'identification des cas cliniques par des campagnes de tests centrés sur les individus). Les tests individuels souffrent donc de biais, car une partie des personnes infectées sont peu ou pas symptomatiques dans le cadre de cette pandémie. Ces tests sont proposés en cas de suspicion d'infection (symptômes et cas-contacts), pour établir certaines autorisations de circulation, ou reposent sur une démarche volontaire qui peut être affectée par les circonstances (nombreux tests réalisés avant les jours fériés, beaucoup moins pendant). Cette démarche conduit à des discontinuités dans les données d'incidence et de positivité. C'est pourquoi les approches épidémiologiques intégrées, à même d'éclairer les pouvoirs publics de façon précoce pour orienter des réponses rapides et adaptées au plus près des territoires, constituent une source d'information complémentaire extrêmement pertinente. Ce type d'approche permet également de suivre certaines mutations spécifiques, évocatrices de variants d'intérêt.

Bien que le SARS-CoV-2 affecte principalement les voies aériennes supérieures, il se réplique également dans I'intestin et est excrété chez près 30 à $60 \%$ des porteurs dans les selles [ZHANG et al., 2020], souvent en grande quantité (mais pas nécessairement sous une forme infectieuse). Ces selles rejoignent les stations d'épuration où le génome viral peut être détecté. La concentration de génome viral dans les eaux usées brutes est donc directement dépendante du nombre de personnes excrétant du virus.

La quantification du génome de SARS-CoV-2 dans les eaux usées s'est donc progressivement imposée comme une approche complémentaire globale, non invasive et peu coûteuse pour suivre la dynamique du virus sur un bassin versant spécifique, correspondant aux habitations et bâtiments dont les égouts débouchent sur la station de traitement des eaux usées contrôlées. La publication par le consortium Obépine d'une étude sur l'île-de-France est l'un des travaux précurseurs mettant en évidence une corrélation entre la quantité de génomes de SARS-CoV-2 dans les eaux usées - mesurée par RT-PCR quantitative (RT-qPCR) - et le nombre de cas dans la population [WURTZER et al., 2020], voir par exemple figure 1. Ces travaux ont eu un important impact en France et à l'étranger.

\subsection{La mise en place du groupement d'intérêt scientifique Obépine}

Le consortium Obépine - projet interdisciplinaire regroupant des équipes de l'Institut français de recherche pour l'exploitation de la mer (Ifremer), de SorbonneUniversité, de l'université de Nancy-Lorraine, de celle de Clermont-Ferrand, de I'Institut de recherche biomédicale des armées (IRBA) et d'Eau de Paris - s'est en effet construit à partir d'avril 2020 autour de cette thématique de suivi des eaux usées. Ce consortium, soutenu financièrement par le ministère de l'Enseignement supérieur, de la Recherche et de l'Innovation (MESRI), a reçu une lettre de mission de la ministre Frédérique Vidal pour mettre sous surveillance les eaux 


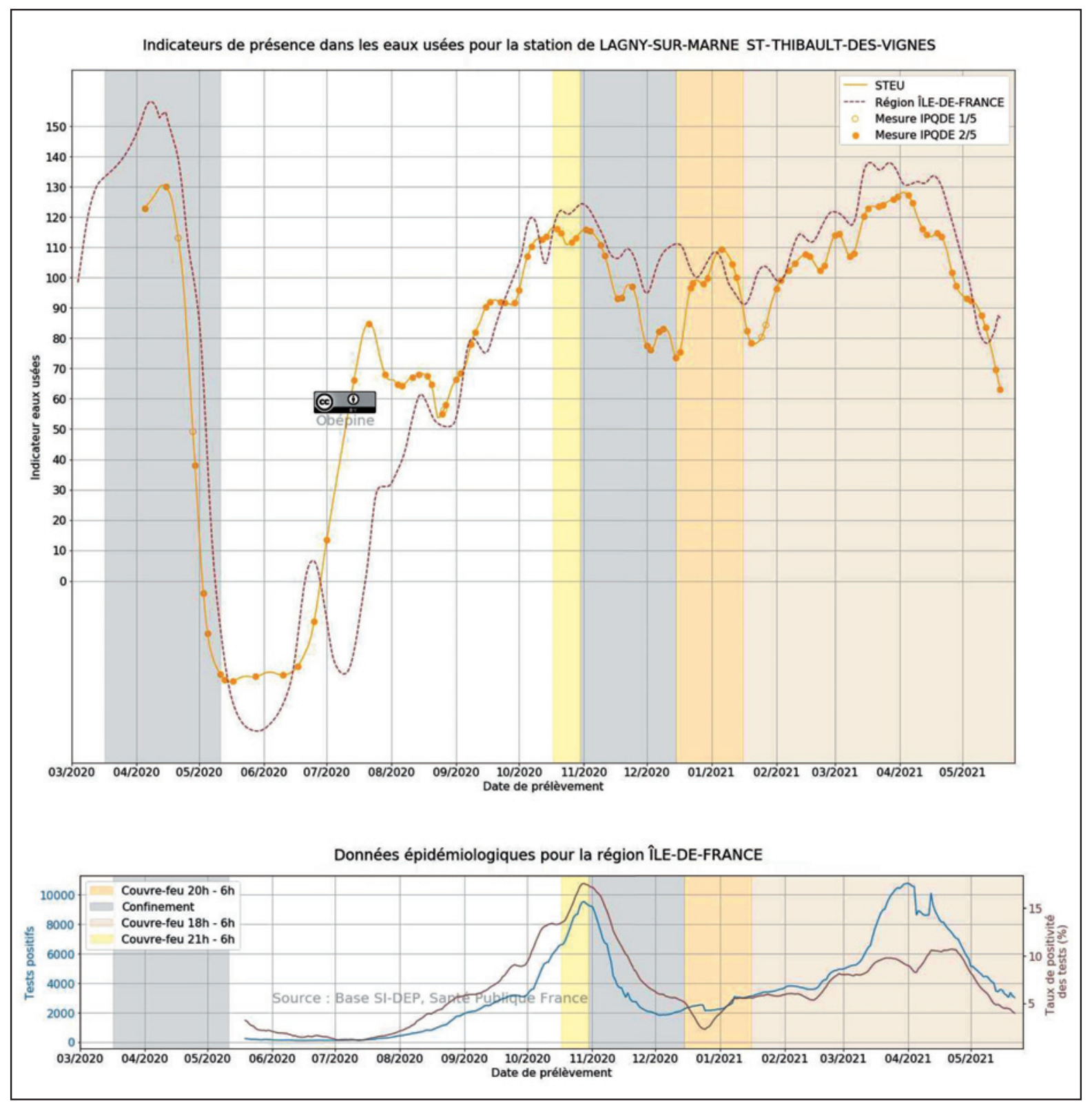

Figure 1. Évolution temporelle de l'indicateur eaux usées (IEU) sur une station d'épuration parisienne. Les données locales (incidence et taux de positivité) confirment que le suivi des eaux usées est un bon reflet de la circulation du virus dans la population. Il faut noter que l'IEU est un indicateur semi-logarithmique, alors que les données épidémiologiques sont représentées sur une échelle linéaire

usées de 150 stations d'épuration sur le territoire national, métropolitain et ultramarin, afin de suivre la dynamique de l'épidémie via la quantification du génome viral dans les eaux usées en juillet 2020.

\section{La mise en place de protocoles fiables et reproductibles}

\subsection{Validation de la stratégie de prélèvements et d'analyses}

Les laboratoires fondateurs du consortium Obépine ont tout d'abord travaillé sur différents sujets techniques et, notamment, sur la validation et la robustesse des pro- tocoles analytiques. Si plusieurs méthodologies étaient proposées dans la littérature scientifique [MEDEMA et al., 2020a], il est rapidement apparu que l'homogénéité et la représentativité des prélèvements étaient essentielles à une interprétation pertinente des quantifications et à leur suivi au cours du temps à l'échelle d'un territoire. En effet, les fluctuations dans les analyses réalisées sur des prélèvements ponctuels ne permettent pas une estimation précise des charges virales moyennes quotidiennes dans les eaux usées des stations. C'est pourquoi au sein du réseau de surveillance piloté par Obépine nous nous sommes attachés à démontrer que 
le prélèvement moyen réalisé sur $24 \mathrm{~h}$ permettait de limiter les fluctuations dues à l'inhomogénéité des eaux usées. La figure 2 illustre les différences importantes que I'on peut observer entre des prélèvements ponctuels et des prélèvements réalisés sur un jour entier. Une erreur d'estimation de 1 à 2 log est ainsi possible - sur la séquence analysée - lorsqu'un prélèvement ponctuel est effectué en lieu et place d'un prélèvement moyen réalisé sur $24 \mathrm{~h}$. Ces résultats ont été confortés par d'autres équipes [AHMED et al., 2021; BIVINS et al., 2021b].

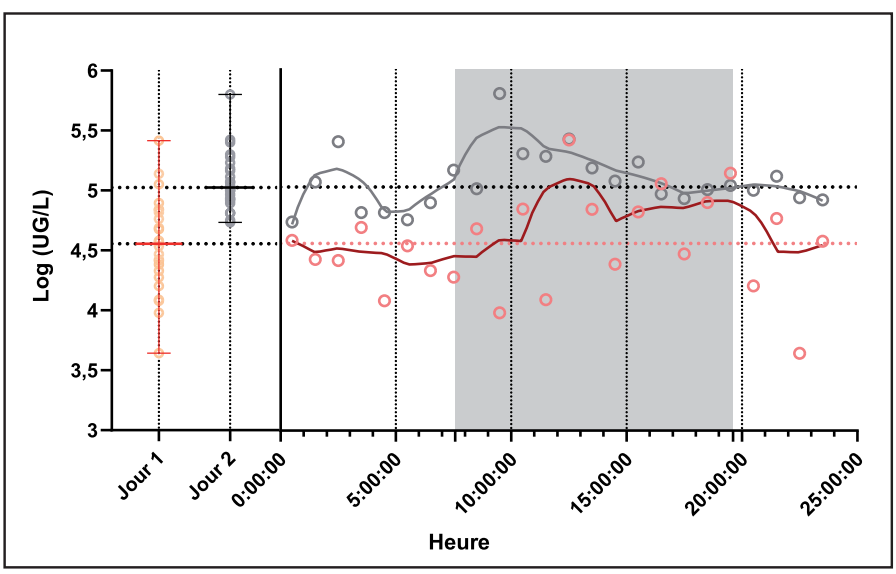

UG/L : unités génomiques/L.

Figure 2. Charges virales SARS-CoV-2 mesurées (par RT-qPCR) sur des prélèvements ponctuels et des prélèvements moyens $(24 \mathrm{~h})$ sur deux collecteurs d'eaux usées. À gauche, répartition des prélèvements individuels sur deux journées; à droite, répartition des points sur l'ensemble de la journée, avec une courbe de la dynamique. La ligne pointillée représente le résultat du prélèvement moyen théorique

Dans un second temps, les équipes d'Obépine ont validé plusieurs techniques de concentration des échantillons. En effet, afin d'améliorer la sensibilité de la méthode, c'est-à-dire la capacité à détecter du génome viral à de faibles concentrations, une étape de concentration de l'échantillon est nécessaire. Pour des raisons à la fois analytiques (forte présence d'inhibiteurs dans les eaux usées) et d'équipement disponible (la technique, pour être largement déployée, doit requérir un minimum d'équipements spécifiques), deux protocoles de concentration et d'extraction ont été définis. Le premier propose une concentration de $11 \mathrm{~mL}$ d'eaux usées [WURTZER et al., 2020] par ultracentrifugation et le second propose une extraction directe sur $5 \mathrm{~mL}$ d'eaux usées [BERTRAND et al., 2021].

La dernière partie de la méthode analytique concerne la quantification du génome viral. Les techniques de RT-qPCR du génome de SARS-CoV-2 dans le cadre du projet Obépine sont basées sur la quantification indépendante d'au moins deux régions du génome viral, dont a minima une RT-qPCR ciblant le gène E. L'expres- sion des résultats se fait en unités génomiques/L (UG/L), en se basant sur une calibration précise. Les plasmides contenant la cible, nécessaires à cette calibration, ont été partagés entre les laboratoires d'analyse du réseau afin de limiter les divergences de quantification.

\subsection{La construction d'un réseau de laboratoires opérationnels}

La mise en place d'un réseau de laboratoires en capacité de réaliser les analyses dans un délai aussi court était une contrainte importante du consortium. Elle a nécessité de nombreux échanges entre les laboratoires de recherche et ceux qui ont rejoint le réseau pour procéder aux analyses. Ces échanges techniques entre les équipes ont rapidement mis en évidence l'intérêt d'organiser des essais interlaboratoires (EIL) au sein des équipes en charge du suivi des stations. Cette homogénéisation des pratiques analytiques était d'autant plus critique que ces mesures - éventuellement complétées par d'autres paramètres - viennent alimenter un modèle mathématique qui convertit les charges virales en un indicateur global de circulation du virus, rendant possible la comparaison des données produites par différents laboratoires et donc de comparer différentes stations entre elles [CLUZEL et al., 2021].

Initialement organisés par les équipes de recherche dans le but de mieux valider leurs protocoles, les EIL sont désormais supervisés, en conformité avec les exigences liées à l'organisation de ce type d'exercice (Norme ISO 13528 : 2015, NF ISO 5725-2 : 2020) par l'Ifremer, l'un des membres d'Obépine. Plus de 25 équipes ont participé au dernier essai, mais seuls 11 laboratoires ont validé l'ensemble des critères imposés permettant de participer au suivi des stations d'épuration. Ce faible taux de réussite s'explique par des difficultés techniques entraînant pour certains participants des dispersions importantes des réplicats, des sensibilités trop faibles ou des faux positifs.

Les résultats des EIL et l'analyse a posteriori de plus de 9000 analyses effectuées dans le cadre du réseau Obépine ont démontré toute l'importance d'un travail de comparaison entre les équipes des laboratoires. Cela a permis d'identifier que certains résultats, notamment dans la gamme des faibles concentrations, doivent être interprétés avec précaution. II est ainsi possible de voir, par exemple dans la figure 3, que deux laboratoires semblent présenter une absence de détection entre leur limite de détection (inférieure à 1000 UG/L pour beaucoup de laboratoires) et environ 20000 UG/L. Les laboratoires suivent des stations de traitement des eaux usées différentes, mais ayant des caractéristiques simi- 


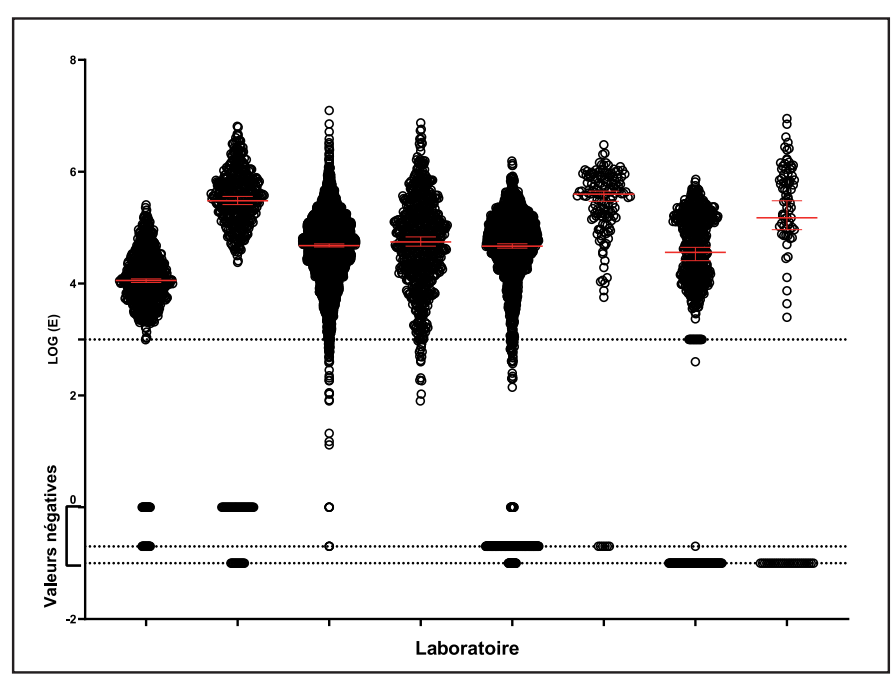

En rouge : valeur médiane et intervalle de confiance à $95 \%$, les lignes pointillées correspondent de haut en bas à la limite de quantification théorique pour $10 \mathrm{~mL}$, puis les valeurs négatives codées différemment en fonction de données internes au laboratoire (absence de signal, résultat aberrant, etc.).

Figure 3. Répartition des quantifications réalisées sur le gène $E$ entre huit laboratoires du réseau, sur la même période, sur des échantillons d'eau usée

laires. On peut observer la distribution spécifique des résultats des laboratoires 2 et 6 qui n'ont jamais obtenu de résultats entre leurs valeurs négatives et leurs plus basses valeurs basses (environ 105).

Néanmoins ces efforts de mise au point au sein de tous les laboratoires ont permis la mise en œuvre d'un suivi analytique bihebdomadaire de plus de 150 stations françaises, avec des méthodes parfois différentes, mais donnant des résultats de dynamique comparable.

\section{Choix des stations...}

Un autre aspect important nécessaire à la construction d'un réseau sentinelle le plus représentatif possible de la situation épidémiologique en France concerne le choix des stations à surveiller. C'est pourquoi à la suite des premières preuves de concept, les équipes d'Obépine ont, avec l'appui des mathématiciens de Sorbonne Université, établi un plan d'échantillonnage afin d'identifier 150 stations (168 actuellement) de référence à partir des 22000 stations françaises. Près de 30 critères - géographiques, économiques, touristiques et démographiques - ont été pris en considération. Les critères choisis concernaient notamment la taille de la population raccordée à la station (des stations de petite taille et des stations liées à la plupart des grandes agglomérations ont été intégrées), l'emplacement géographique (une station au moins par département), la typologie urbaine (présence d'hôpitaux, d'universités, d'activités touristiques...). Des critères techniques individuels ont modifié la liste initiale des stations choisies (station récemment rénovée, difficulté d'accessibilité, etc.) et ont permis d'inclure des stations qui n'étaient pas ciblées initialement. La carte nationale de ces stations dites « mères » suivies est présentée dans la figure 4. Par ailleurs, 10 stations présentant des caractéristiques similaires ont été associées à chaque station mère pour adapter le réseau au gré de l'évolution spatio-temporelle de l'épidémie sur le territoire.

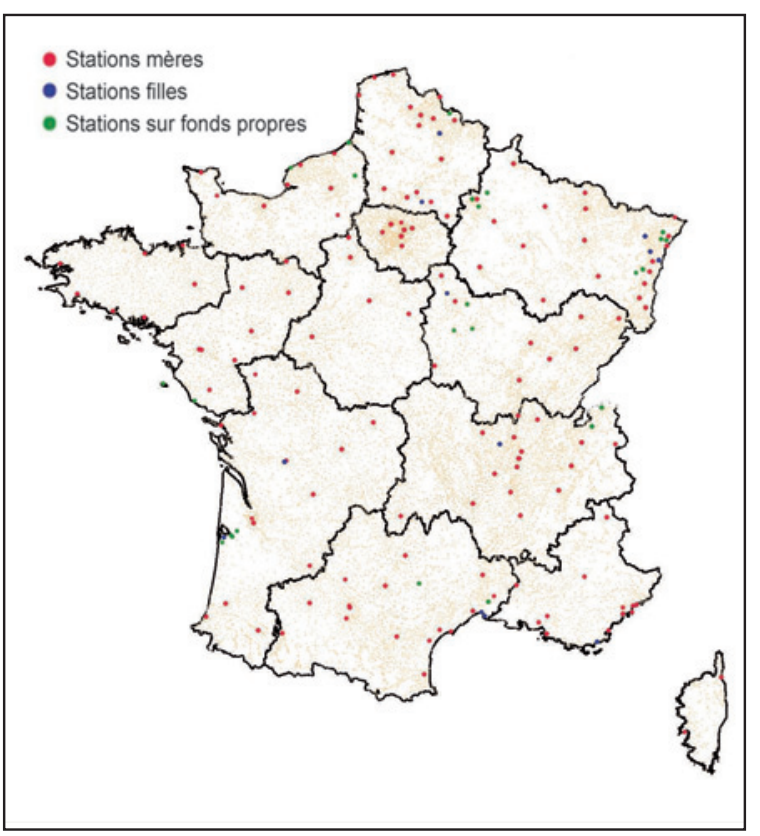

Figure 4. Carte nationale des stations d'épuration suivies dans le cadre du réseau Obépine

\section{La mise en place d'indicateurs indépendants des méthodes analytiques}

Les données brutes de quantification virale (charge virale par litre d'eau usée au jour J) sont bruitées de par la nature même des protocoles analytiques malgré toutes les précautions prises pour limiter cette variation [BIVINS et al., 2021a]. Divers paramètres (débit des stations, informations de gestions hydrauliques des réseaux, paramètres chimiques...) sont intégrés afin de corriger au mieux ces fluctuations. Après avoir été collectées dans une base centralisée, ces données sont traitées par un modèle mathématique permettant de lisser les écarts (filtre de Kalman backward/forward), d'intégrer l'effet des précipitations, et produire in fine un indicateur de circulation semi-logarithmique, sans unité, permettant de comparer les niveaux de circulation du virus d'un territoire à l'autre (figure 1) et de fait d'éclairer les pouvoirs publics sur la circulation du virus sur le territoire. Cet indicateur développé spécifiquement est présenté dans deux publications [CLUZEL et al., 2021 ; COURBARIAUX et al., 2021]. 
Cet indicateur permet de suivre la dynamique locale. II met en évidence, de façon très bien corrélée avec les données d'incidence, une augmentation, une stabilité ou une diminution de la concentration en génome viral et permet de «qualifier » la situation locale ou régionale (circulation faible, moyenne, forte) (figure 5). Une cartographie nationale de la dynamique virale observée dans les eaux usées est également possible.

\section{Des données accessibles au plus grand nombre}

Très rapidement, une démarche d'ouverture des données s'est mise en place avec le concours du MESRI. Après transmission aux agences régionales de santé (ARS) et aux collectivités locales les données de l'indicateur sont mises à disposition du public sous forme de cartes et courbes de tendance locales ${ }^{8}$. Les valeurs de l'indicateur sont aussi transférées dès obtention sur la plateforme de données publiques du gouvernement ${ }^{9}$ contribuant de ce fait à un large partage par le consortium des données produites et corrigées à l'attention des autorités de santé, des scientifiques et des citoyens.

${ }^{8}$ www.reseau-obepine.fr

${ }^{9} \mathrm{https} / / /$ www.data.gouv.fr/fr/datasets/surveillance-du-sars-cov-2-dans-leseaux-usees-1

\section{Des résultats précoces et pertinents}

La démonstration d'une relation entre concentrations en virus dans les eaux usées et données épidémiologiques individu-centrées a été confortée - outre par les travaux princeps d'Obépine - par de nombreuses équipes [MEDEMA et al., 2020b], faisant pour la première fois, sans doute à cette échelle, de l'épidémiologie des eaux usées une véritable aide aux politiques sanitaires publiques. En s'appuyant sur les analyses d'eaux usées, un réseau opérationnel de surveillance de l'épidémie Covid-19 a été mis en place en France et s'est avéré utile pour suivre la circulation de l'épidémie et suivre l'efficacité des mesures de gestion prises.

En effet, les travaux réalisés dès le 20 mars 2020 en îlede-France avaient apporté une remarquable démonstration de l'efficacité des mesures de confinement strictes sur la circulation virale : le génome viral était devenu indétectable dans les eaux usées des cinq stations suivies dès la première semaine de mai 2020 Ce suivi quantitatif a également permis d'identifier des remontées précoces dans les eaux usées et de détecter la seconde vague en Île-de-France dès le 23 juin 2020, alors que les indicateurs individu-centrés n'y ont montré d'évolution que fin juillet au plus tôt. L'avance du signal " eaux usées » sur les indicateurs syndromiques (formes symptomatiques de Covid-19) dépend probablement

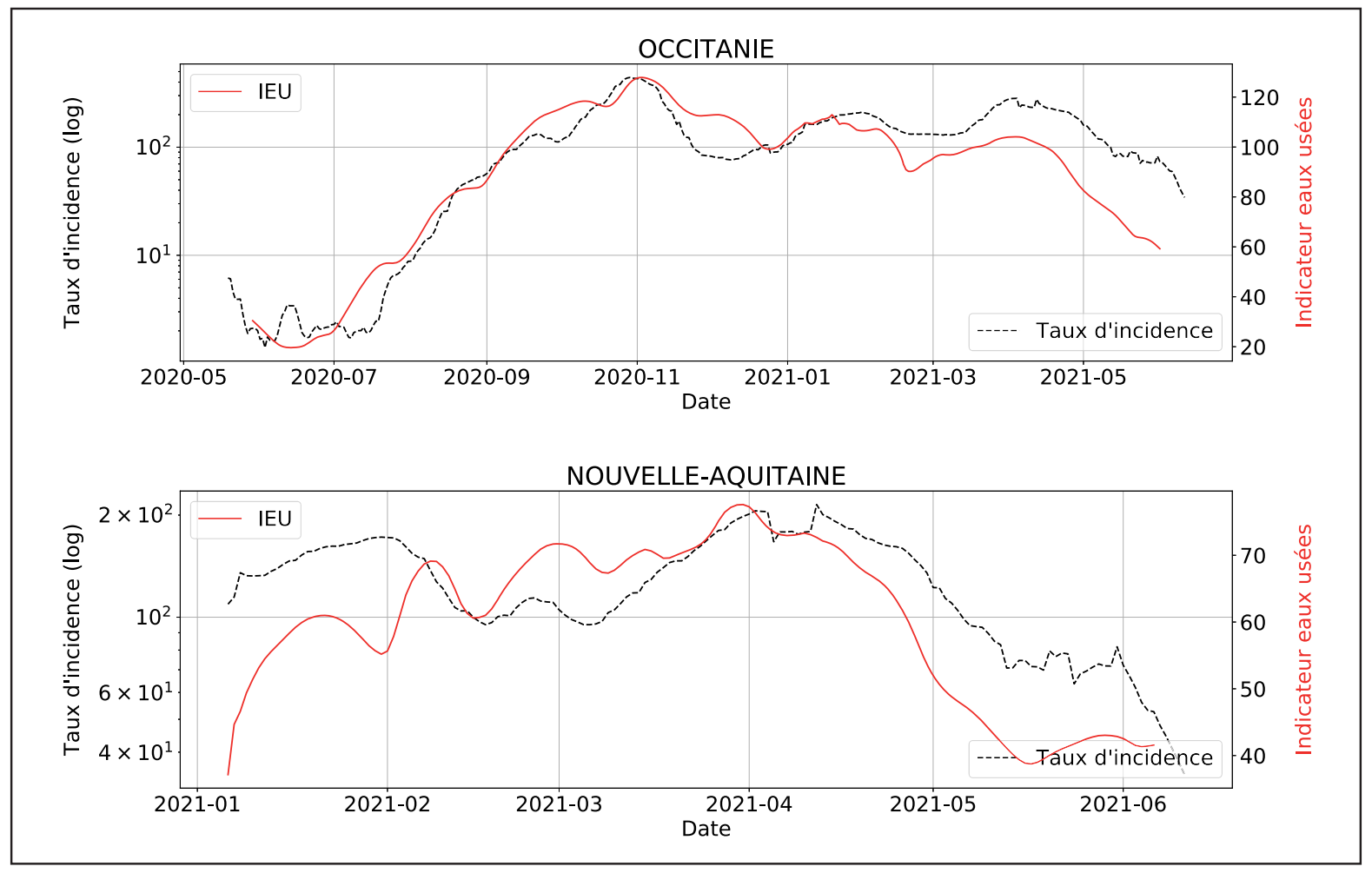

IEU : indicateur eaux usées.

Figure 5. Exemple de la dynamique régionale au regard des indicateurs sanitaires d'incidence régionaux 
de la sensibilité des personnes infectées à la maladie (rarement exprimée chez les sujets jeunes sans facteurs de risque) et du niveau de surveillance des populations dans les campagnes de tests massifs (RT-PCR et tests antigéniques). Ces éléments nous laissent supposer que l'avance remarquable du signal " eaux usées » sur les indicateurs traditionnels - durant l'été 2020 - est liée sans doute au fait que la recirculation virale s'est d'abord opérée chez des sujets jeunes, peu sensibles aux formes cliniques puis, dès la rentrée, chez des sujets plus âgés ou fragiles, et a entraîné ainsi la dégradation tardive des indicateurs. Les analyses réalisées par Obépine ont également permis de démontrer l'impact des mesures de freinage collectives : effet remarquable du confinement de mars 2020 - comme nous l'avons déjà souligné -, effet plus modéré de la séquence couvre-feu/confinement modéré dans laquelle certains territoires sont entrés depuis octobre 2020. Dans les eaux usées, la troisième vague de mars 2021 apparaît davantage comme une reprise de la seconde, en grande partie liée à l'introduction d'un variant plus contagieux (variant Alpha, dit « anglais »).

Des actions de recherche sur le SARS-CoV-2, notamment sur l'infectiosité du SARS-CoV-2 dans les selles, les eaux usées, les boues, sur la bioconcentration par les mollusques bivalves, l'état du virus dans l'environnement et, plus récemment, sur la recherche des variants viraux ont également été mis en place au sein de ce groupement.

\section{Perspectives}

Plus récemment et devant le risque d'émergence de variants plus contagieux, plus virulents et potentiellement capables d'échapper en partie à la réponse immunitaire, un suivi spécifique s'est mis en place. Couplant la recherche ciblée de certaines mutations associées à certains variants à des approches globales de « métagénomique » (analyse sans a priori des variations génétiques des virus dans les eaux usées), le

\section{Bibliographie}

AHMED W., BIVINS A., BERTSCH P.M., BIBBY K., GYAWALI P., SHERCHAN S.P., SIMPSON S.L., THOMAS K.V., VERHAGEN R., KITAJIMA M., MUELLER J.F., KORAJKIC A. (2021) : « Intraday variability of indicator and pathogenic viruses in $1-h$ and $24-h$ composite wastewater samples: Implications for wastewaterbased epidemiology ». Environmental Research ; 193 : 110531. BERTRAND I., CHALLANT J., JEULIN H., HARTARD C., MATHIEU L., LOPEZ S., SCHVOERER E., COURTOIS S., GANTZER C., (2021) : "Epidemiological surveillance of SARS-CoV-2 by genome quantification in wastewater applied to a city in the consortium est aujourd'hui en mesure de proposer un plan de surveillance pour les variants connus (ou à venir) sur l'ensemble du territoire français métropolitain grâce à des enquêtes flash pouvant être réalisées, par exemple, une à deux fois par mois ou en intégrant un suivi quantitatif régulier de certaines mutations évocatrices [WURTZER et al., 2021b]. Cette démarche semble particulièrement pertinente à l'heure où l'on s'interroge sur l'impact de la vaccination sur la circulation des virus " classiques » autant que sur la sélection de variants plus résistants aux anticorps neutralisants résultant d'une infection naturelle ou induits par la vaccination. Dans un contexte préoccupant d'émergence des variants dits Delta et Delta+, potentiellement plus contagieux que le variant Alpha, le risque de réinfection des sujets déjà infectés naturellement a été évoqué. L'entrée dans l'été 2021 s'est accompagnée d'une réduction des gestes barrières et d'une augmentation des contacts sociaux exposant de fait les jeunes majoritairement non vaccinés à maintenir un réservoir viral qui pourrait donner lieu à une nouvelle flambée à l'automne 2021.

L'épidémiologie basée sur les eaux usées est ainsi un outil important en santé publique pour plusieurs raisons : elle rend possible 1) une détection précoce de la circulation d'un agent infectieux; 2) un suivi global d'une épidémie, indépendamment des politiques de dépistage ou des classes d'âge des populations infectées, permettant une visualisation de sa dynamique au cours du temps; 3) une recherche locale de clusters sous réserve d'une bonne maîtrise du prélèvement; et enfin, 4) grâce à des qPCR spécifiques, ou des séquençages ciblés, le suivi de variants. C'est pourquoi la mise en place pérenne d'un observatoire sentinelle des eaux usées, proposé par le consortium Obépine dès avril 2020 - associant recherche multidisciplinaire et veille sanitaire - constituerait une avancée majeure pour détecter précocement et suivre les épidémies et pour limiter leurs impacts sanitaires, économiques et sociaux.

northeast of France: Comparison of ultrafiltration- and protein precipitation-based methods ». International Journal of Hygiene and Environmental Health ; 233 : 113692.

BIVINS A., KAYA D., BIBBY K., SIMPSON S.L., BUSTIN S.A., SHANKS O.C., AHMED W. (2021a) : «Variability in RT-qPCR assay parameters indicates unreliable SARS-CoV-2 RNA quantification for wastewater surveillance ». Water Research ; 203 : 117516.

BIVINS A., NORTH D., WU Z., SHAFFER M., AHMED W., BIBBY K. (2021b) : «Within-Day Variability of SARS-CoV-2 RNA in 
municipal wastewater influent during periods of varying Covid-19 prevalence and positivity [preprint] ». Epidemiology. DOI : 10.1101/2021.03.16.21253652

CLUZEL N., COURBARIAUXM., WANG S., MOULIN L., WURTZER S., BERTRAND I., LAURENT K., MONTFORT P., OBEPINE CONSORTIUM, LE GUYADER S., BONI M., MOUCHEL J.-M., MARÉCHAL V., NUEL G., MADAY Y. (2021) : « Mathematical modeling and adequate environmental sampling plans are essential for the public health assessment of Covid-19 pandemics: development of a monitoring indicator for SARS-CoV-2 in wastewater ». Submitted. DOI : 10.1101/2021.09.01.21262877

COURBARIAUX M., CLUZEL N., WANG S., MARÉCHAL V., MOULIN L., WURTZER S., OBEPINE CONSORTIUM, MOUCHEL J.-M., MADAYY., NUEL G. (2021) : « An autoregressive model for a censored data denoising method robust to outliers with application to the Obépine SARS-CoV-2 monitoring ». arXiv:2108.02115 [stat].

MEDEMA G., BEEN F., HEIJNEN L., PETTERSON S. (2020a) : " Implementation of environmental surveillance for SARS-CoV-2 virus to support public health decisions: Opportunities and challenges ». Current Opinion in Environmental Science \& Health ; 17, 49-71.

MEDEMA G., HEIJNEN L., ELSINGA G., ITALIAANDER R., BROUWER A. (2020b) : « Presence of SARS-Coronavirus-2 RNA in sewage and correlation with reported Covid-19 prevalence in the early stage of the epidemic in The Netherlands ». Environmental Science \& Technology Letters ; $7: 511-6$.

MIURA T., LHOMME S., LE SAUX J.-C., LE MEHAUTE P., GUILLOIS Y., COUTURIER E., IZOPET J., ABRANAVEL F., LE GUYADER F.S. (2016) : " Detection of hepatitis $E$ virus in sewage after an outbreak on a French island ». Food and Environmental Virology ; 8(3) : 194-9.
PREVOST B., LUCAS F.S., AMBERT-BALAY K., POTHIER P. MOULIN L., WURTZER S. (2015a) : « Deciphering the diversities of astroviruses and noroviruses in wastewater treatment plant effluents by a high-throughput sequencing method ». Applied and Environmental Microbiology ; 81(20) : 7215-22.

PREVOST B., LUCAS F.S., GONCALVES A., RICHARD F., MOULIN L., WURTZER S. (2015b) : « Large scale survey of enteric viruses in river and waste water underlines the health status of the local population ». Environmental International ; 79 : 42-50.

WURTZER S., MARÉCHAL V., MOUCHEL J.-M., MADAY Y., TEYSSOU R., RICHARD E., ALMAYRAC J.-L., MOULIN L. (2020) : « Evaluation of lockdown effect on SARS-CoV-2 dynamics through viral genome quantification in waste water, Greater Paris, France, 5 March to 23 April $2020 »$. Eurosurveillance 25, 2000776. DOI : 10.2807/1560-7917.ES.2020.25.50.2000776

WURTZER S., MARÉCHAL V., BERTRAND I., BONI M., LE GUYADER S., MOULIN L., MADAY Y., GANTZER C., MOUCHEL J.-M. (2021a) : « Maladies infectieuses virales vues au travers des eaux usées ». Virologie ; 25(1) : 8-11. DOI : 10.1684/vir.2021.088

WURTZER S., WALDMAN P., LEVERT M., MOUCHEL J.M., GORGÉ O., BONI M., MADAY Y., OBÉPINE CONSORTIUM, MARÉCHAL V., MOULIN L. (2021b) : « Monitoring the propagation of SARS CoV2 variants by tracking identified mutation in wastewater using specific RT-qPCR [preprint] ». Infectious Diseases. DOI : 10.1101/2021.03.10.21253291

ZHANG Y., CHEN C., SONG Y., ZHU S., WANG D., ZHANG H., HAN G., WENG Y., XU JUN, XU JIANAN, YU P., JIANG W. YANG X., LANG Z., YAN D., WANG Y., SONG J., GAO G.F., WU G., XU W. (2020) : «Excretion of SARS-CoV-2 through faecal specimens ». Emerging Microbes \& Infections ; 9(1) : 2501-8. 


\section{Assainissement \\ Catalogue des publications}
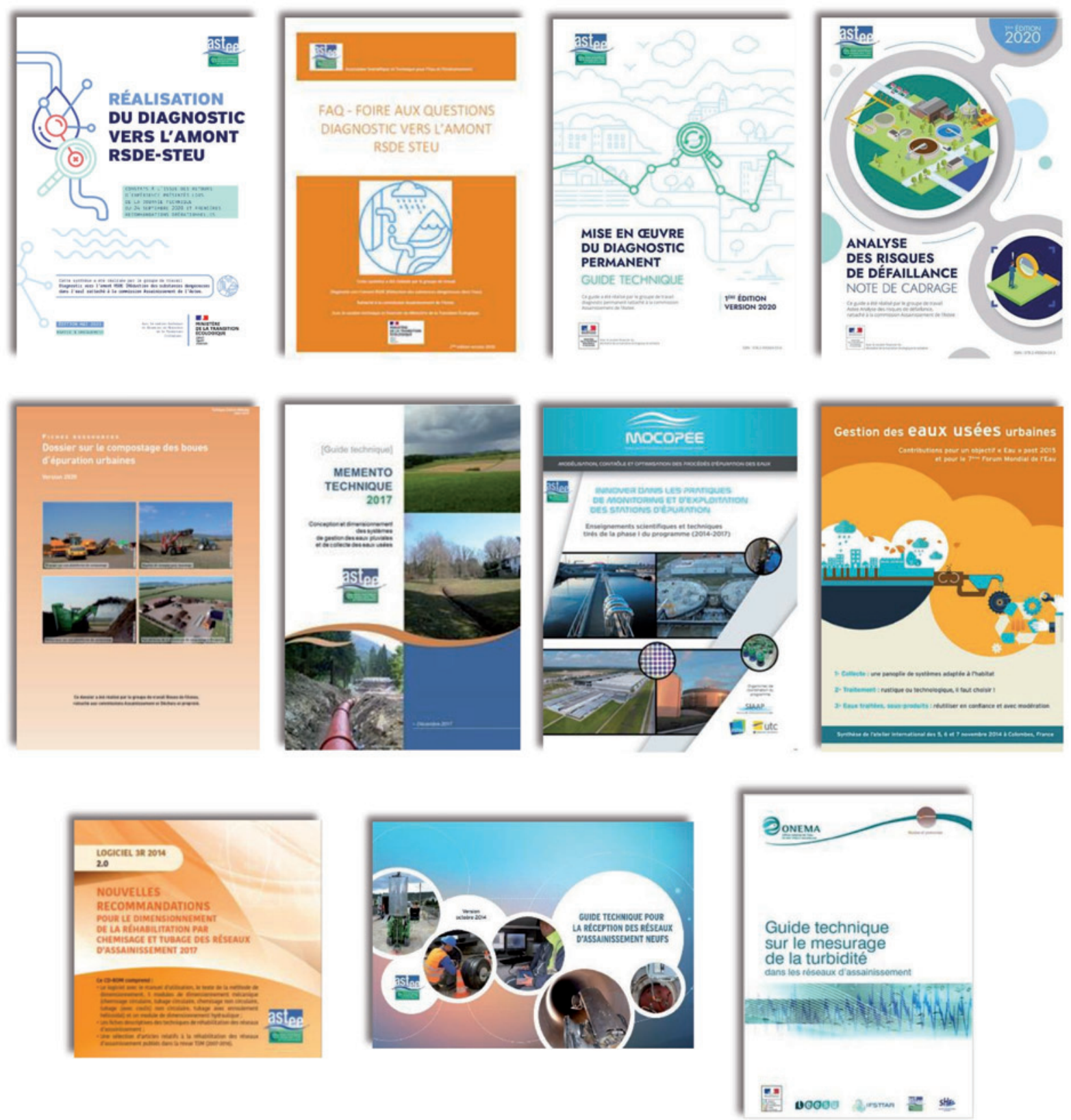

Créée en 1905

Reconnue d'utilité publique $\mathbf{3} \mathbf{8 0 0}$ membres 


\section{Tous les appels à contributions TSM}

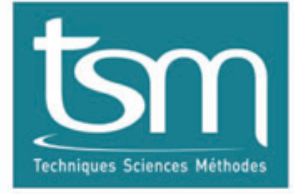

\section{QUALITÉ DES EAUX}

Plastiques et microplastiques

20 décembre 2021

PRÉVENTION ET VALORISATION DES DÉCHETS

Valorisation des biodéchets dans les territoires

11 janvier 2022
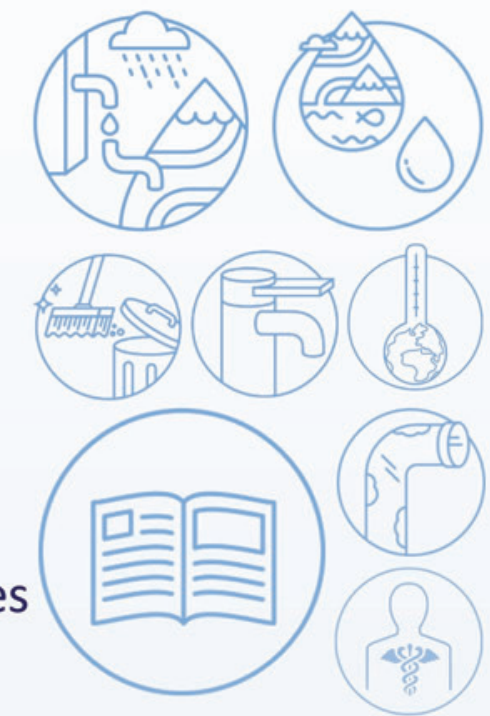

GESTION PATRIMONIALE - URBANISME

Diagnostic permanent : connaître et maintenir ses infrastructures

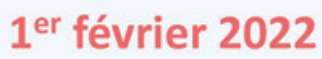

\section{PROCÉDÉS ET EXPLOITATION}

Plan de gestion des risques dans le domaine de l'eau $1^{\text {er }}$ mars 2022

\section{ET CONTRIBUEZ, VOUS AUSSI, AU NUMÉRO SPÉCIAL « CRISE SANITAIRE LIÉE À LA COVID »}

Des études scientifiques et techniques aux retours d'expérience sur la gestion de crise ou la continuité de service...

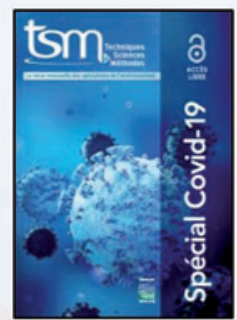

Réception des articles en continu, numéro en accès libre et complété au fil de l'eau ! ?

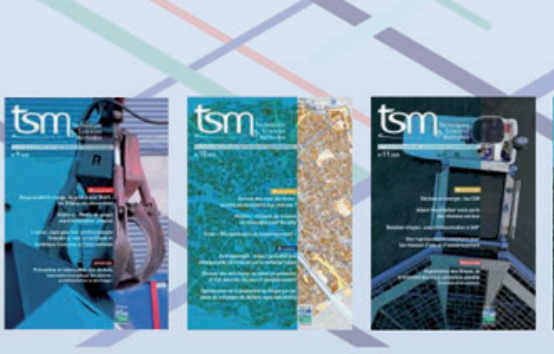

Plus d'informations sur astee-tsm.fr 